\title{
COCHRANE
} CORNER

${ }^{\dagger}$ This review is an abridged version of a Cochrane Review previously published in the Cochrane Database of Systematic Reviews, 2014, Issue 7, doi: 10.1002/14651858.CD009268. pub2 (see www.thecochranelibrary. com for information). Cochrane Reviews are regularly updated as new evidence emerges and in response to feedback, and the Cochrane Database of Systematic Reviews should be consulted for the most recent version of the review.

We thank the Cochrane Review Group for their support in publishing these reviews

\section{Haloperidol versus low-potency first-generation antipsychotic drugs for schizophrenia}

Magdolna Tardy, Maximilian Huhn, Werner Kissling, Rolf R. Engel, Stefan Leucht

\section{Background}

Antipsychotic drugs are the core treatment for schizophrenia. Treatment guidelines state that there is no difference in efficacy between antipsychotic compounds, however, lowpotency antipsychotic drugs are often clinically perceived as less efficacious than high-potency compounds, and they also seem to differ in their side-effects.

\section{Objectives}

To review the effects in clinical response of haloperidol and low-potency antipsychotics for people with schizophrenia.

Search methods

We searched the Cochrane Schizophrenia Group Trials Register (July 2010).

\section{Selection criteria}

We included all randomised trials comparing haloperidol with first-generation low-potency antipsychotic drugs for people with schizophrenia or schizophrenia-like psychosis.

\section{Data collection and analysis}

We extracted data independently. For dichotomous data, we calculated risk ratios (RR) and their $95 \%$ confidence intervals (CI) on an intention-to-treat basis based on a random-effects model. For continuous data, we calculated mean differences $(\mathrm{MD})$, again based on a random-effects model.

\section{Main results}

The review currently includes 17 randomised trials and 877 participants. The size of the included studies was between 16 and 109 participants. All studies were short-term with a study length between 2 and 12 weeks. Overall, sequence generation, allocation procedures and blinding were poorly reported. We found no clear evidence that haloperidol was superior to low-potency antipsychotic drugs in terms of clinical response (haloperidol 40\%, low-potency drug 36\%, 14 RCTs, $n=574$, $\mathrm{RR}=1.11, \mathrm{Cl} 0.86$ to 1.44 , low-quality evidence). There was also no clear evidence of benefit for either group in acceptability of treatment, with equivocal difference in the number of participants leaving the studies early for due to any reason (haloperidol 13\%, low-potency antipsychotics 17\%, 11 RCTs, $n=408, \mathrm{RR}=0.82, \mathrm{Cl} 0.38$ to 1.77 , low-quality evidence). Similar equivocal results were found between groups for experiencing at least one adverse effect (haloperidol 70\%, low-potency antipsychotics $35 \%, 5$ RCTs, $n=158, \mathrm{RR}=1.97$, Cl 0.69 to 5.66 , very low-quality evidence). More participants from the lowpotency drug group experienced sedation (haloperidol 14\%, low-potency antipsychotics 41\%, 2 RCTs, $n=44, R R=0.30, \mathrm{Cl}$ 0.11 to 0.82 , moderate quality evidence), orthostasis problems (haloperidol 25\%, low-potency antipsychotics $71 \%, 1$ RCT, $n=41, \mathrm{RR}=0.35, \mathrm{Cl} 0.16$ to 0.78 ) and weight gain (haloperidol $5 \%$, low-potency antipsychotics $29 \%, 3 \mathrm{RCTs}, n=88, \mathrm{RR}=0.22$, $\mathrm{Cl} 0.06$ to 0.81 ). In contrast, the outcome 'at least one movement disorder' was more frequent in the haloperidol group (haloperidol $72 \%$, low-potency antipsychotics $41 \%, 5$ RCTs, $n=170, \mathrm{RR}=1.64, \mathrm{Cl} 1.22$ to 2.21, low-quality evidence). No data were available for death or quality of life. The results of the primary outcome were robust in several subgroup and sensitivity analyses.

\section{Authors' conclusions}

The results do not clearly show a superiority in efficacy of haloperidol compared with low-potency antipsychotics. Differences in adverse events were found for movement disorders, which were more frequent in the haloperidol group, and orthostatic problems, sedation and weight gain, which were more frequent in the low-potency antipsychotic group. The quality of studies was low, and the quality of evidence for the main outcomes of interest varied from moderate to very low, so more newer studies would be needed in order to draw a definite conclusion about whether or not haloperidol is superior or inferior to low-potency antipsychotics.

Assessed as up to date: 19 May 2012

See more at: http://summaries.cochrane.org/CD009268/SCHIZ_ haloperidol-versus-first-generation-low-potency-antipsychotic-drugs-forschizophrenia 